\title{
Novel Treatments for Multiple Organ Failure: A Brief Account of Enthusiasms, Disappointments and Neglect
}

\author{
Giorgio Noera ${ }^{1 *}$ and Alfio Bertolini ${ }^{2}$ \\ ${ }^{1}$ Maria Cecilia Hospital, GVM Care \& Research Cardiac Surgery Unit, Italy \\ ${ }^{2}$ Late Professor and Chairman, Department of Medicine and Division of Clinical Pharmacology, School of Medicine of the University of \\ Modena and Reggio Emilia, Italy
}

*Corresponding author: Giorgio Noera, Maria Cecilia Hospital, GVM Care \& Research Cardiac Surgery Unit, Italy

\section{ARTICLE INFO}

Received:

Published: 幽 August 31, 2020

Citation: Giorgio Noera, Alfio Bertolini. Novel Treatments for Multiple Organ Failure: A Brief Account of Enthusiasms, Disappointments and Neglect. Biomed J Sci \& Tech Res 29(5)-2020. BJSTR. MS.ID.004870.

\section{Introduction}

Exposure to all causes that trigger shock status are marked out by immunological unique time-related host response against inadequate oxygen delivery to tissues. The consequently multiple organ dysfunction syndrome (MODS) and the multi organ failure (MOF) are marked out by time-related host response from a lifethreatening status to death. Nowadays, the causality is a most common but least recognized disease where the basically unchanged mortality reflects a real unmet medical need. Haemorragic, or anyway hypovolemic, traumatic, endotoxic, septic shock, massive fluid losses as in extensive burns, acute heart failure, polytrauma they are characterized by acute endothelipathy and occurrence of disseminated intravascular coagulation, that doubles mortality rate in severe trauma [1]. Together with undernutrition and, on the contrary, obesity-related pathologies, infectious diseases, and malignancies, MOF represents one of the leading causes of mortality worldwide. Despite the tremendous social impact and despite the huge number of experimental and clinical studies, the progress in both prevention and treatment of this dramatic clinical condition has been very limited.
Some conceptually innovative first-aid treatments have been proposed during the last decades, either with -at the end- disappointing results following an initial enthusiasm or with incredulity and non-consideration.

\section{Essentials of Pathophysiology of Multiple Organ Failure}

The tissue hypoperfusion/hypoxia triggers, if not promptly corrected (hopefully within minutes: "platinum ten minutes"; at least within the first hour: "golden hour"), an overamplified cascade of events that ultimately leads to multiple organ failure and death (organ failure in shock involves damage to remote organs that are not part of the initial injury: for example, intestinal ischemia is followed by failure of the lungs, heart, brain and other organs ). The above-mentioned cascade of events comprises the pooling of blood (or of the residual blood, in the case of massive hemorrhage) in the capillaries of peripheral tissues and the consequent tissue hypoxia [ the massive release of opioid peptides plays a role of key importance: opioids inhibit sympathetic outflow and noradrenaline release from sympathetic terminals and contribute to peripheral 
pooling of blood, decreased venous return, and reduced cardiac output (for review see: [2])]. In turn, hypoxia triggers an unrestrained, disproportionate systemic inflammatory response ("systemic inflammatory response syndrome", SIRS) that starts within the first minutes and is the principal cause of the ensuing tissue damage [3], together with the overamplification of the complement and coagulation cascades [4].

A key initiating role in such inflammatory cascade is played by the activation of the ubiquitary early transcription factor NF-kB (nuclear factor $\mathrm{kB}$ ), of mitogen-activated protein-kinases (MAPKs), and by the accumulation of oxygen and nitrogen free radicals. The activated NF-kB triggers the transcription of hundreds target genes in the nucleus and the ensuing production of several factors of the inflammatory response: cell adhesion molecules, inducible nitric oxide (NO) synthase (iNOS), chemokines, cytokines (in particular TNF-alpha) and cytokine receptors. TNF-alpha plays a fundamental role in the pathophysiology of shock: it inhibits noradrenaline release from sympathetic terminals, induces hyporeactivity of hearth and vessels to noradrenaline, exerts a myocardial depressant effect, and further stimulates iNOS with consequent massive overproduction of NO, which is one of the main responsibles for the hemodynamic decompensation and vascular hyporeactivity that occur after massive hemorrhage and in septic shock [for review see: [2]].

Exposure to traumatic or infectious insults results in a rapid involvement of the complement cascade, with an increase in circulating complement activation products, such as $\mathrm{C} 3 \mathrm{a}, \mathrm{C} 5 \mathrm{a}$, and $\mathrm{sC} 5 \mathrm{~b}$ 9; an enhanced $\mathrm{C} 3 \mathrm{a} / \mathrm{C} 3$ ratio in plasma early after trauma being prognostic for lethal outcome [4]. Particularly after severe traumas there is moreover a release of inflammasomes, intracellular multiprotein complexes (mainly NLRP3, NLRP1, and AIM2) that activate caspases, that in turn allow the release of mature forms of IL-1beta and IL-18 and trigger cell death. A role of increasingly recognized importance in the overall, complex "scenario" of MOF pathophysiology is played by the endothelium (see for reviews: [5]). In adult humans, endothelium has a surface area of approximately $5000 \mathrm{~m}^{2}$ and forms the innermost lining of all blood and lymphatic vessels. Under normal conditions the endothelium is anticoagulated by a number of anticoagulant systems including the negatively charged luminal surface layer, the glycocalix. The endothelial glycocalix is composed of sulphated glycoproteins connected with sialic acids (heparan sulphate, dermatan sulphate), proteoglycans, and non-sulphated glycosaminoglycans. It is extremely vulnerable to damage (such as in septic shock, major trauma, ischemia-reperfusion), and plays a role in clinical deterioration of the patient, up to multiorgan failure. The intestine, too, plays a central role in multiorgan failure [6].

When the mucosal epithelial barrier becomes permeable, as in shock, the digestive enzymes escape into the wall of the intestine, digest tissues in the mucosa thus generating cytotoxic fragments, enter into the systemic circulation and activate other degrading proteases. There is a tight relationship between lactate levels and the late phase development of MOF. Hyperlactacydemia is associated with poor outcome of shock and reflects inadequate tissue perfusion. This has placed lactate in the center of guiding resuscitation. However, given the complex metabolism and clearance of lactate, its actual use is complex [7]. By using other markers of microcirculation status and of tissue perfusion together with lactate levels provides a more solid framework to guide the initial hours of resuscitation [8]. Persistent microcirculatory derangement in association with hyperlactacydemia may reflect ongoing shock and long-lasting damage even in the setting of hemodynamic compensation ("occult shock"). Also, the serum concentration of IL- 6 within the first $24 \mathrm{~h}$ after trauma is useful for the prediction of post-traumatic Multiple Organ Failure/Multiple Organ Dysfunction syndrome (MOF/MOD syndrome) and mortality [9].

\section{Therapeutic Options for Multiple Organ Failure}

"There is currently no effective treatment for multiorgan failure following shock other than alleviation supportive care". This statement is probably too pessimistic; however, it is true that the best treatment for MOF is represented by measures aimed at preventing its development. The therapeutic protocols for the care of shock basically dictate rather obvious measures aimed at tissue reperfusion/reoxygenation : whole blood, freeze-dried plasma (plasma has replaced crystalloid for volume expansion in traumatic hemorrhagic shock), cathecholamines (norepinephrine the first line in vasodilatory shock, after adequate volume restoration), inotropes (dobutamine the first line)(dopamine is recommended only in bradycardic patients), vasopressin, angiotensin II (in distributive shock, including septic shock), vit. C, thiamine, corticosteroids, antibiotic therapy, mechanical circulatory support (MCS) devices (in cardiogenic shock), etc.: nothing really innovative. On the whole, guidelines and drugs for the treatment of shock and to prevent the development of multiple organ failure are remained practically the same during the last decades ("...there remains no ideal standard of care for shock patients"). As a consequence, the prognosis, too, is remained basically the same, as well as the incidence of mortality [10].

Yet, some innovative ideas (few indeed) have been put forward during the last forty years, supported by animal data and by pilot clinical studies (these last not always confirmed, however). The first innovative idea came from Holaday, Faden and associates $[11,12]$. When it became apparent that during shock there is a massive activation of opioid systems , they suggested an important pathogenetic role of endorphins, and demonstrated that in fact, in animal models, the opiate antagonist naloxone reversed hypovolemic, endotoxic, and other kinds of shock (its efficacy being however attenuated by acidosis and hypothermia, and cardiac arrhythmias being a possible complication). But human data were far from uni- 
vocal. Moreover, too high doses of naloxone were required (up to dozen times higher than the life-saving ones used for the reversal of opiate overdose; and hence with not negligible side effects, and increased pain awareness) Thus, after an initial optimism regarding the clinical efficacy of opiate antagonists in shock, this novel, initially promising way, has been abandoned [13-16].

The second innovative idea came from Bertolini and coworkers and was supported by the results obtained in animal and human conditions of hemorrhagic shock [17-21].

Bertolini and coworkers were accumulating experimental data in support of the idea, shared with other groups [22] that melanopeptides (peptides of the MSH/ACTH family) and some opioid peptides (essentially $\beta$-endorphin), both derived from the same precursor (proopiomelanocortin, POMC), play the role of mutual physiological antagonists in many fundamental functions of the body, and constitute a system of basic importance for the general homeostasis of the organism. When Holaday, Faden and coworkers produced their data demonstrating that endo-opioids are massively released in conditions of tissue hypoxia and might play an important role in the pathogenesis of shock, as initially suggested by the first results obtained with naloxone, Bertolini and coworkers wanted to see whether melanopeptides, too, could reverse a shock condition, as a further confirmation of their proposed role of endogenous antagonists of opioids. The experiments - performed in anesthetized and heparinized rats and dogs, stepwise bled to a condition of Wiggers type of hemorrhagic shock without reinfusion, invariably lethal 25-30 min after bleeding termination showed that melanopeptides [ACTH and several ACTH fragments, including alpha-MSH and other fragments almost totally or totally at all devoid of corticotrophic activity] i.v. bolus injected within 10 min after bleeding, actually induce a dose-dependent reversal of hypotension and of respiratory depression, and the restoration of cardiac output.

The increase in the volume of circulating blood being the consequence of the mobilization of the residual peripherally pooled, "out of currency" blood [capillary pooling and trapping of blood -"capillary paralysis"- characterize the terminal phase of shock]. The maximum active doses of the most potent ACTH fragments (80-160 micrograms/kg in the case of the sequences 1-24, 1-18, 1-17, 1-16, 1-13) completely/almost completely restored arterial pressure, pulse amplitude and respiratory rate to pre-bleeding values, and produced a $100 \%$ survival (without reinfusion of the shed blood ) for more than $24 \mathrm{~h}$. Adrenal glands are not involved, because the effect is the same either in intact or in adrenalectomized animals, and is obtained to the same degree either with ACTH fragments with full adrenocorticotropic activity or with fragments practically devoid of such activity. As said above, the reversal of such condition of hemorrhagic shock is associated with the mobilization of the residual blood, trapped in capillaries and in large blood reservoirs (liver, spleen).
Indeed, the reversal of the shock condition and the restoration of arterial and pulse pressure, which take place within a few minutes after the injection of melanocortin peptides, are associated with an impressive increase both in the volume of circulating blood and in the tissue blood flow, and are largely prevented by splenectomy or by the ligature of the suprahepatic veins [23]. Moreover, there is a gradual and almost complete recovery (within $60 \mathrm{~min}$ ) of venous $\mathrm{PO} 2, \mathrm{PCO} 2$, and SO2. The normalization of blood $\mathrm{pH}$ and lactate - which is however alike complete $60 \mathrm{~min}$ after treatment- is on the other hand preceded by a further initial worsening, probably due to the mobilization of the residual blood that had been trapped in hypoxic tissues. This effect of melanocortins was confirmed by other groups and in other animal models of shock: hypovolemic shock produced in rabbits by the graded occlusion of the inferior vena cava [24-28]; splanchnic ischemia/reperfusion-induced shock in rats; and also, in a pre-terminal condition produced in rats by prolonged asphyxia. The melanocortin-induced shock reversal prevents the development of MOF, as demonstrated by histological and immunocytochemical examination of heart, lung, liver and kidney tissues $24 \mathrm{~h}$ after treatment, either in rats or in a specific mice model of multiple organ dysfunction syndrome [29-32].

In hemorrhage-shocked animals treated with a maximum effective dose of melanocortin within 5-10 min after hemorrhage, reinfusion of the shed blood definitively reverses shock and cures a substantial number of animals even if performed 30,60, or even 120 min later. These animal data prompted to extend the investigations to humans. In clinical studies, the melanocortin ACTH-(1-24) has been used, because marketed from several years for some clinical conditions - thus easily available also in off-label pathologies- and completely devoid of acute toxicity up to the dose of $100 \mathrm{mg}$ in adult subjects. After some anecdotal case reports showing that the i.v. bolus injection of 5-10 mg of ACTH-(1-24), in addition to the conventional therapeutic protocol, produced a dramatic improvement in extremely critical conditions (rupture of the right atrium, massive bleeding from oesophageal varices, dissection of aortic aneurism, etc.) [33], a clinical trial enrolling 32 subjects, all with type A aortic dissection complicated by aortic rupture and cardiac tamponade, and with clinical signs of severe shock, confirmed the life-saving effect of ACTH-(1-24)(survival one month after surgery: $87 \%$ in the ACTH group, $47 \%$ in the standard treatment group) [34].

Moreover, a randomized phase 3 clinical study conducted on 100 critically ill in shock patients, exposed to emergency surgery with expected mortality $>20 \%$, has shown that in treated with high doses of bolus tetracosactide, zeroing the mortality at 30 days towards $18.8 \%$ of the untreated and the normalization of the cytokine overload within 18 hours after surgery [35]. In spite of such exciting clinical results, confirming so many experimental data obtained by different groups in a variety of animal conditions of tissue hypoxia; in spite of the fact that such life-saving treatment is safe, simple, easy to administer also "in the field" ; in spite of the fact 
that these data have been published in the most authoritative and circulated medical journals, and described in many international congresses ; in spite of all that, clinicians have ignored this novel therapeutic approach.

The third innovative idea came from Tracey and coworkers. Following the demonstration by Watkins, et al. [36], Goehler, et al. [37] of a vagus-visceral chemosensory pathway, communicating the immune system with brain structures [= inflammatory molecules produced in damaged tissues activate afferent signals that are carried by ascending vagus nerve fibers (and by other pain and ascending sensory pathways): vagus neurons express IL-1 receptor mRNA [38] and vagotomy blunts the development of fever in animals i.p. injected with IL-1] , Borovikova, et al. [39], Tracey, et al. [40] demonstrated that the stimulation of efferent vagus fibers attenuates the systemic inflammatory response to endotoxin: the acetylcholine released by vagus terminals in organs of the reticuloendothelial system (especially liver, spleen, gastrointestinal tract, heart) interacts with alpha7-nicotinic receptors in tissue macrophages, which inhibits the release of pro-inflammatory cytokines (TNF-alpha, IL-1 alpha, IL-1beta, IL-6 and IL-18), but not of anti-inflammatory cytokines (as IL-8 and IL-10 ). So, a vagus-centered "cholinergic anti-inflammatory pathway", or "inflammatory reflex" [41] exists, that is triggered in real time by inflammatory molecules.

However, it is insufficient to effectively prevent an unrestrained, shock-producing, inflammatory process. Only its augmentation (by electrical stimulation of efferent vagus fibers or by pharmacological activation of alpha7-nicotinic receptors) making it possible to counteract exacerbated pro-inflammatory cytokine release. [It seems worth mentioning that several years before the discovery of the "cholinergic anti-inflammatory pathway " by Goehler and Watkins and by Tracey and colleagues, the group of Bertolini et al. had described that their extreme experimental condition of hemorrhagic shock in rats was temporarily reversed by the i.v. injection of nicotine, or of dimethylphenylpiperazinium [42-44], two prototypic agonists at nicotinic receptors]. Many animal data have confirmed the existence of the cholinergic anti-inflammatory pathway, and the protective effect of its stimulation in different experimental conditions of tissue hypoxia: endotoxic shock, hemorrhagic shock, sepsis, myocardial ischemia/reperfusion, intestinal ischemia/reperfusion, renal ischemia/reperfusion (for reference [44]).

As an example, in a condition of otherwise rapidly lethal hemorrhagic shock in rats, electrical stimulation of efferent vagus nerves reverted the extremely severe hypotension, increased survival time, blunted liver NF-kB activation, decreased hepatic TNF-alpha mRNA and plasma TNF-alpha levels. Such effects of vagal stimulation being prevented by pretreatment with chlorisondamine, a nicotinic receptor antagonist [45]. As another example, in a murine model of polymicrobial sepsis, treatment with nicotine or with the alpha7-nicotinic receptor selective agonist GTS-21 (anabaseine) for 3 days produced a significant survival improvement $[46,47]$. This effect being associated with suppression of the release of HMGB1 (high mobility group box 1 protein, a late mediator of inflammation following the earlier "cytokine storm" caused by TNF-alpha and other early inflammatory mediators). Again, just to quote only some examples: in a rat model of myocardial ischemia/reperfusion injury, electrical stimulation of efferent vagal fibers strongly reduced the high incidence of severe arrhythmias and lethality, the increase in free radical blood levels, and myocardial histologic alterations, while enhancing the antiapoptotic prosurvival kinase ERK $1 / 2$ activation.

These effects being prevented by atropine, thus suggesting that, in the case of myocardium, muscarinic -instead of nicotinic- acetylcholine receptors are involved [48]. Administration of nicotine or GTS-21 had protective effects also in rat models of renal ischemia-reperfusion injury [49], of intestinal ischemia-reperfusion injury, and of cerulein-induced pancreatitis in mice. On the other hand, neither vagus nerve stimulation nor nicotinic agonist treatment suppress the pro-inflammatory cytokine release in the lungs [50-52]. But, contrary to animal experiments, the (very few indeed) so far published clinical studies have produced rather inconsistent results [51,52]. Moreover, vagus stimulation implies two main shortcomings:

(i) It produces obvious, not negligible, foreseeable, negative effects, particularly on cardiovascular and respiratory functions: decreased heart rate, bronchoconstriction, significant reduction of oxygen saturation (in the $87-100 \%$ of patients); due to the activation of pulmonary and cardio-inhibitory vagal fibers;

(ii) It requires a not so simple surgical preparation: obviously, this is hardly conceivable in dramatically pressing situations in casualty wards or even in the field.

Thus, at present, either invasive or non-invasive vagus electrical stimulation is used for the chronic treatment of other diseases: rheumatoid arthritis, Crohn's disease, refractory epilepsy, depression, painful conditions such as cluster migraine ; with usually positive results, albeit with some methodological uncertainties [53-56].

\section{Discussion and Conclusions}

Ideally, a treatment aimed at preventing the development of multiple organ failure should be:

a) (Obviously) effective,

b) Of simple, easy and rapid execution,

c) Devoid ofimportant side effects.

In the course of the last 40 years, essentially three really innovative treatments have been proposed, on the basis of original ideas and of novel experimental data. The treatment with naloxone, and more in general with opiate antagonists, in the idea that a massive 
release of endogenous opioids plays a central role in the pathogenesis of shock and in its catastrophic slipping into multiple organ failure, was initially rather enthusiastically endorsed, also in view of the results in animal studies, but was at the end abandoned, in the light of several inconsistencies (active doses manifold higher than those required for the blockade of opiates, with consequent disturbing side effects; disappointing clinical results ). The vagus nerve stimulation, in order to magnify the cholinergic anti-inflammatory pathway, was alike initially unanimously welcomed, particularly in view of the results obtained in animal models of shock, in laboratory setting.

But it turned out to be practically unfeasible in humans, especially out of hospital, "on the scene", as in case of labour or traffic accidents: isolation of the vagus nerve requires time, delicate surgical manipulations, skilled operators, time. The treatment with melanocortins (in practice with ACTH 1-24, tetracosactide) has proven effective not only in many different animal models of shock, but in human conditions as well, and has many advantages: (i) Absence of toxicity following a single, i.v. bolus injection of the maximum effective dose of $10 \mathrm{mg}$ (ACTH 1-24 has been i.v. injected up to the dose of $100 \mathrm{mg}$ in adults with no adverse event ; only if administered repeatedly at short intervals it may cause an excessive production of aldosterone by the adrenals, with consequent liquid retention : it is imperative that ACTH 1-24be "bolus"-injectedin order to reach immediately the maximum concentration in the body, and hence the maximum effect);

(ii) Extreme easiness of administration (single i.v. bolus injection);

(iii) Multitargeted mechanism of action, which involves brain structures, vagus nerve, sympathetic terminals,immune cells, balance of the opioid/anti-opioid system, with prevalence of the anti-opioid (melanocortinergic) arm (not simply blockade of the endogenous opioids, as in the case of naloxone,but effects opposite to those of opioids); etc. (Table 1);

Table 1: Mechanisms involved in the effect of melanocortins in conditions of tissue hypoperfusion-hypoxia, either of the whole body (shock, respiratory arrest, cardiac arrest) or of single organs (heart, brain, intestine, kidney, etc.

\begin{tabular}{|c|c|c|c|c|}
\hline Track & Trigger & System & Response & Effect \\
\hline $\begin{array}{l}\text { Hypoxia } \\
\text { Injury } \\
\text { Ischemia }\end{array}$ & $\begin{array}{c}\text { MC1 \& MC3 } \\
\text { Immunocite melanocor- } \\
\text { tin receptors }\end{array}$ & NF-kB & $\begin{array}{l}\text { Inhibition of massive production of } \\
\text { several factors of the inflammatory } \\
\text { response (cell adhesion molecules, } \\
\text { chemokines, cytokines and cytokines } \\
\text { receptors) }\end{array}$ & $\begin{array}{l}\text { Highly significant reduction of free radicals in } \\
\text { blood; decrease of liver TNF-alpha mRNA and of } \\
\text { plasma levels of TNF-alpha }\end{array}$ \\
\hline & & Inducible NOS & Normalization of NO blood levels & Reduced expression of adhesion molecules. \\
\hline & $\begin{array}{l}\text { MC4 receptors in the } \\
\text { dorsal motor nucleus of } \\
\text { the vagus }\end{array}$ & $\begin{array}{l}\text { Efferent vagus } \\
\text { fibers }\end{array}$ & $\begin{array}{l}\text { Activation of the descending "choliner- } \\
\text { gic anti-inflammatory pathway" }\end{array}$ & $\begin{array}{l}\text { Acetylcholine release in organs of the reticu- } \\
\text { loendothelial system (especially liver, spleen, } \\
\text { gastrointestinal tract, heart) }\end{array}$ \\
\hline & & $\begin{array}{l}\text { Alpha7-nico- } \\
\text { tinic receptors } \\
\text { on tissue } \\
\text { macrophages }\end{array}$ & $\begin{array}{l}\text { Inhibition of the production of } \\
\text { pro-inflammatory cytokines (TNF-al- } \\
\text { pha, IL-1alpha, IL-6, IL-1beta, and } \\
\text { inflammatory mediators of the early } \\
\text { "cytokine storm". }\end{array}$ & $\begin{array}{l}\text { Stimulation of the production of anti-inflamma- } \\
\text { tory cytokines (IL-8, IL-10). } \\
\text { Defunctionalization of primary afferent } \\
\text { substance P-containing nerve fibers), or of a } \\
\text { Substance P antagonist. } \\
\text { Functional antagonism against endogenous } \\
\text { opioids ( opioids inhibit sympathetic outflow and } \\
\text { noradrenaline release from sympathetic termi- } \\
\text { nals and inflammatory reflex). }\end{array}$ \\
\hline
\end{tabular}

(iv) Almost immediate, temporary restoration of cardiovascular and of respiratory functions, which allows to extend the "platinum ten minutes", so to reach a casualty wardstill in recoverable conditions.

In view of the above facts, we are of the opinion (in point of fact, we are firmly convinced) that ACTH 1-24 bolus injection should be included in the first aid treatment protocol of shock and of any clinical condition at risk of shock.

\section{References}

1. Gangloff C, Mingant F, Theron M, Galinat H, Grimault O, et al. (2019) New considerations on pathways involved in acute traumatic coagulopathy: the thrombin generation paradox. World J Emerg Surg 12: 14-57.
2. Leone S, Noera G. Bertolini A (2013) Melanocortins as innovative drugs for ischemic diseases and neurodegenerative disorders: established data and perspectives. Curr Med Chem 20(6): 735-750.

3. Chakraborty RK, Burns B (2019) Systemic inflammatory response syndrome. Stat Pearls.

4. Karasu E, Nilsson B, Kohl J, Lambris JD, Huber Lang M (2019) Targeting complement pathways in polytrauma- and sepsis-induced multiple organ dysfunction. Front Immunol 10: 543-560.

5. Johansson P, Stensballe J, Ostrowski RS (2017) Shock induced endotheliopathy (SHINE) in acute critical illness - a unifying pathophysiologic mechanism Critical Care 21: 25.

6. Altshuler AE, Kistler EB, Schmid Schonbein GW (2016) Autodigestion: proteolytic degradation and multiple organ failure in shock. Shock 45(5): 483-489. 
7. Astapenko D, Benes J, Pouska J, Lehmann C, Islam S, et al. (2019) Endothelial glycocalix in acute care surgery - what anaesthesiologists need to know for clinical practice. BMC Anesthesiology 19(1): 238-261.

8. Bakker J, Postelnicu R, Mukherjee V (2020) Lactate: where are we now? Crit Care Clin 36(1): 115-124.

9. Qiao Z, Wang W, Yin L, Luo P, Greven J, et al. (2018) Using IL-6 concentrations in the first $24 \mathrm{~h}$ following trauma to predict immunological complications and mortality in trauma patients: a metaanalysis. Eur J Trauma Emerg Surg 44(5): 679-687.

10. Simmons J, Ventetuolo CE (2017) Cardiopulmonary monitoring of shock Curr. Opin Crit Care 23(3): 223-231.

11. Holaday JW, Faden AI (1978) Naloxone reversal of endotoxic hypotension suggests role of endorphins in shock. Nature 275: 450-451.

12. Faden AI, Holaday JW (1979) Opiate antagonists: a role in the treatment of hypovolemic shock. Science 205(4403): 317-318.

13. Gurll NJ, Reynolds DG, Vargish T, Lechner R (1982) Naloxone without transfusion prolongs survival and enhances cardiovascular function in hypovolemic shock. J Pharm Exp Ther 220: 621-624.

14. Bonnet F, Bilaine J, Lhoste F, Mankikian B, Kerdelhue B, et al. (1985) Naloxone therapy of human septic shock. Crit Care Med 13(11): 972975.

15. Allolio B, Fischer H, Kaulen D, Deuss U, Winkelmann W (1987) Naloxone in treatment of circulatory shock resistant to conventional therapy. Klin Wochenschr 65: 213-217.

16. Reynolds DG, Gurll NJ, Holaday JW, Lechner RB (1989) The therapeutic efficacy of opiate antagonists in hemorrhagic shock. Resuscitation 18(23): $243-251$.

17. Bertolini A, Guarini S, Ferrari W (1986) Adrenal-independent anti-shock effect of ACTH-(1-24) in rats. Eur J Pharmacol 122: 387-388.

18. Bertolini A, Guarini S, Rompianesi E, Ferrari W (1086) $\alpha$-MSH and other ACTH fragments improve cardiovascular function and survival in experimental hemorrhagic shock. Eur J Pharmacol 130(1-2): 19-26.

19. Bertolini A, Guarini S, Ferrari W, Noera G, Massini C, et al. (1987) ACTH (1-24) restores blood pressure in acute hypovolaemia and haemorrhagic shock in humans. Eur J Clin Pharmacol 32(5): 537-538.

20. Bertolini A, Ferrari W, Guarini S (1989) The adrenocorticotropic hormone $(\mathrm{ACTH})$-induced reversal of hemorrhagic shock. Resuscitation 18(2-3): 253-267.

21. Noera G, Lamarra M, Guarini S, Bertolini A (2001) Survival rate after early treatment for acute type-A aortic dissection with ACTH-(1-24). Lancet 358(9280): 469-470.

22. Bertolini A, Ferrari W (1982) Evidence and implications of a melanocortins-endorphins homeostatic system. In Endroczi E (Edn.): Neuropeptides and psychosomatic processes. Akadémiai Kiadò , Budapest, pp. 245-261.

23. Ludbrook J, Ventura S (1995) ACTH-(1-24) blocks the decompensatory phase of the haemodynamic response to acute hypovolaemia in conscious rabbits. Eur J Pharmacol 275(3): 267-275.

24. De Wied D, Jolles J (1982) Neuropeptides derived from pro-opiocortin: behavioral, physiological and neurochemical effects. Physiol Rev 62(3): 976-1059.

25. Gispen WH, Van Ree JM, de Wied D (1977) Lipotropin and the central nervous system. Int Rev Neurobiol 20: 201-250.

26. Jacquet YF (1978) Opiate effects after adrenocorticotropin or $\beta$-endorphin injection in the periaqueductal gray matter of rats. Science 201(4360): 1032-1034.

27. O'Donohue TL, Dorsa DM (1982) The opiomelanotropinergic neuronal and endocrine systems. Peptides 3(3): 353-395.
28. Bazzani C, Tagliavini S, Bertolini E, Bertolini A, Guarini S (1992) Influence of ACTH-(1-24) on metabolic acidosis and hypoxemia induced by massive hemorrhage in rats. Resuscitation 23(2): 113-120.

29. Squadrito F, Guarini S, Altavilla D, Squadrito G, Campo GM, et al. (1999) Adrenocorticotropin reverses vascular dysfunction and protects against splanchnic artery occlusion shock. Br J Pharmacol 128(3): 816-822.

30. Guarini S, Bazzani C, Bertolini A (1997) Resuscitating effect of melanocortin peptides after prolonged respiratory arrest. $\mathrm{Br}$ J Pharmacol 121(7): 1454-1460.

31. Giuliani D, Mioni C, Bazzani C, Zaffe D, Botticelli Ar, et al. (2007) Selective melanocortin MC4 receptor agonists reverse haemorrhagic shock and prevent multiple organ damage. Br J Pharmacol 150(5): 595-603.

32. Bitto A, Polito F, Altavilla D, Irrera N, Giuliani D, et al. (2011) Melanocortins protect against multiple organ dysfunction syndrome in mice. Br J Pharmacol 162(4): 917-928.

33. Mandel W, Singer MJ, Gudmondson HR, Meister L, Modern FW (1951) Intravenous use of pituitary adrenocorticotropic hormone (ACTH): a report on its administration in twenty-five patients. JAMA 146(6): 546551.

34. Noera G, Lamarra M, Guarini S, Bertolini A (2001) Survival rate after early treatment for acute type-A aortic dissection with ACTH-(1-24). Lancet 358(9280) : 469-470.

35. Noera G, Camerino C, Ghetti L, Calzà L, Picano E, et al. (2015) Melanocortin adrenocorticotropic anti-opioid peptide tetracosactide improves survival in high- risk patients undergoing cardiac surgery: a single center, randomized, placebocontrolled, phase III study. European Society of Cardiology Congress 2015 Best Poster Session 1.

36. Watkins LR, Goehler le, Relton JK, Tartaglia N, Silbert L, et al. (1995) Blockade of interleukin-1-induced hyperthermia by subdiaphragmatic vagotomy: evidence for vagal mediation of immune-to-brain communication. Neurosci Lett 183(1-2): 27-31.

37. Goehler LE, Relton JK, Dripps D, Kiechle R, Tartaglia N, et al. (1997) Vagal paraganglia bind biotinylated interleukin-1 receptor antagonist: a possible mechanism for immune-to-brain communication. Brain Res Bull 43(3): 357-364.

38. Goehler LE, Gaykema RP, Hansen MK, Anderson K, Maier SF, et al. (2000) Vagal immune-to-brain communication : a visceral chemosensory spathway. Auton Neurosci 85(1-3): 49-59.

39. Borovikova LV, Ivanova S, Zhang M, Yang H, Botchkina GI, et al. (2000) Vagus nerve stimulation attenuates systemic inflammatory response to endotoxin. Nature 405: 458-462.

40. Tracey KJ (2002) The inflammatory reflex. Nature 420(6917): 853-859.

41. Pavlov VA, Tracey KJ (2006) Controlling inflammation: the cholinergic anti-inflammatory pathway. Biochem Soc Trans 34(Pt 6): 1037-1040.

42. Guarini S, Tagliavini S, Bazzani C, Bertolini A, Ferrari W (1991) Nicotine reverses hemorrhagic shock in rsats. Naunyn Schmiedeberg's Arch Pharmacol 343: 427-430.

43. Guarini s, Bazzani C, Tagliavini S, Bertolini A, Ferrari W (1992) Reversal of experimental hemorrhagic shock by dimethylphenylpiperazinium (DMPP). Experientia 48: 663-667.

44. Bertolini A (2012) Drug-induced activation of the nervous control of inflammation: a novel possibility for the treatment of hypoxic damage. Eur J Pharmacol 679(1-3): 1-8.

45. Guarini S, Altavilla D, Cainazzo MM, Giuliani D, Bigiani A, et al. (2003) Efferent vagus fibre stimulation blunts nuclear factor -kappaB activation and protects against hypovolemic hemorrhagic shock. Circulation 107: $1189-1194$

46. Wang H, Liao H, Ochani M, Justiniani M, Lin X, et al. (2004) Cholinergic agonists inhibit HMGB1 release and improve survival in experimental sepsis. Nat Med 10(11): 1216-1221. 
47. Pavlov VA, Ochani M, Yang LH, Lin X, Levi J, et al. (2007) Selective alpha7nicotinic acethylcholine receptor agonist GTS-21 improves survival in murine endotoxemia and severe sepsis. Crit Care Med 35(4): 1139-1144.

48. Mioni C, Bazzani C, Giuliani D, Altavilla D, Leone S, et al. (2005) Activation of an efferent cholinergic pathway produces strong protection against myocardial ischemia/reperfusion injury in rats. Crit Care Med 33(11): 2621-2628

49. Yeboah MM, Xue X, Duan B, Ochani M, Tracey Kj, et al. (2008) Cholinergic agonists attenuate renal ischemia-reperfusion injury in rats. Kidney Int $74(1): 62-69$

50. Pavlov VA (2008) Cholinergic modulation of inflammation. Int J Clin Exp Med 1(3): 203-212.

51. Bernik TR, Friedman SG, Ochani M, Di Raimo R, Ulloa L, et al. (2002) Pharmacological stimulation of the cholinergic anti-inflammatory pathway. J Exp Med 195(6): 781-788.

\section{ISSN: 2574-1241}

DOI: $10.26717 /$ BJSTR.2020.29.004870

Giorgio Noera. Biomed J Sci \& Tech Res

(C) This work is licensed under Creative

Submission Link: https://biomedres.us/submit-manuscript.php
52. Van Westerloo DJ, Giebelen IA, Florquin S, Bruno MJ, Larosa GJ, et al. (2006) The vagus nerve and nicotinic receptors modulate experimental pancreatitis severity in mice. Gastroenterology 130(6): 1822-1830.

53. Kox M, Van Eijk LT, Verhaak T, Frenzel T, Kiers HD, et al. (2015) Transvenous vagus nerve stimulation does not modulate the innate immune response during experimental endotoxemia: a randomized controlled study. Arthritis Research and Therapy 17: 150.

54. Lerman I, Hauger R, Sorkin L, Proudfoot J, Davis B, et al. (2016) Noninvasive transcutaneous vagus nerve stimulation decreases wholeblood culture-derived cytokines and chemokines: a randomized, blinded, healthy control pilot trial. Neuromodulation 19(3): 283-290.

55. Yuan H, Silberstein SD (2016) Vagus nerve and vagus nerve stimulation, a comprehensive review: part II. Headache 56(2): 259-266.

56. Burger AM, Verkuil B (2018) Transcutaneous nerve stimulation via the tragus: are we really stimulating the vagus nerve? Brain Stimul 11(4): 945-946.

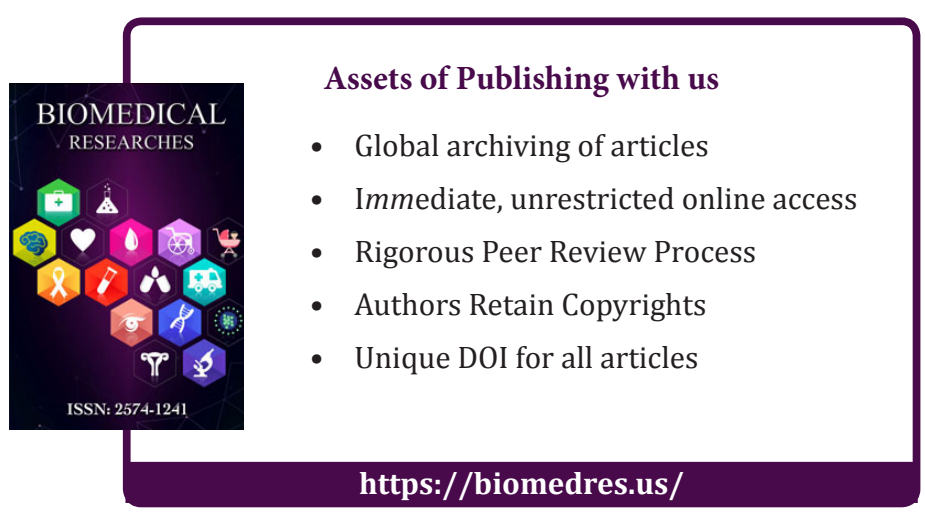

